\title{
THEMIS ground-space observations during the development of auroral spirals
}

\author{
A. Keiling ${ }^{1}$, V. Angelopoulos ${ }^{2}$, J. M. Weygand ${ }^{2}$, O. Amm ${ }^{3}$, E. Spanswick ${ }^{4}$, E. Donovan ${ }^{4}$, S. Mende ${ }^{1}$, J. McFadden ${ }^{1}$, \\ D. Larson ${ }^{1}$, K.-H. Glassmeier ${ }^{5}$, and H. U. Auster ${ }^{5}$ \\ ${ }^{1}$ Space Sciences Laboratory, University of California, Berkeley, CA, USA \\ ${ }^{2}$ Institute of Geophysics and Planetary Physics, University of California, Los Angeles, CA, USA \\ ${ }^{3}$ Finnish Meteorological Institute, Helsinki, Finland \\ ${ }^{4}$ Department of Physics and Astronomy, University of Calgary, Calgary, Canada \\ ${ }^{5}$ Institut of Geophysics and extraterrestrial Physics, TU Braunschweig, Germany
}

Received: 21 May 2009 - Revised: 26 October 2009 - Accepted: 30 October 2009 - Published: 27 November 2009

\begin{abstract}
A simultaneous observation of an auroral spiral and its generator region in the near-Earth plasma sheet is rather unlikely. Here we present such observations using the THEMIS spacecraft as well as the THEMIS ground network of all-sky imagers and magnetometers. Two consecutive auroral spirals separated by approximately $14 \mathrm{~min}$ occurred during a substorm on 19 February 2008. The spirals formed during the expansion phase and a subsequent intensification, and were among the brightest features in the aurora with diameters of 200-300 km. The duration for the formation and decay of each spiral was less than $60 \mathrm{~s}$. Both spirals occurred shortly after the formation of two oppositely rotating plasma flow vortices in space, which were also accompanied by dipolarizations and ion injections, at $\sim 11 R_{E}$ geocentric distance. Observations and model calculations also give evidence for a magnetic-field-aligned current generation of approximately $0.1 \mathrm{MA}$ via the flow vortices, connecting the generator region of the spirals with the ionosphere, during the formation of both spirals. In the ionosphere, a pair of equivalent ionospheric current (EIC) vortices with opposite rotations (corresponding to upward and downward currents) was present during both auroral spirals with enhanced EICs and ionospheric flows at the locations of the auroral spirals and along the auroral arcs. The combined ground and space observations suggest that each auroral spiral was powered by two oppositely rotating plasma flow vortices that caused a current enhancement in the substorm current wedge.
\end{abstract}

Keywords. Magnetospheric physics (Auroral phenomena; Magnetosphere-ionosphere interactions; Plasma sheet)

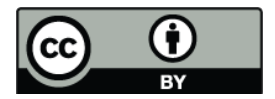

Correspondence to: A. Keiling (keiling@ @sl.berkeley.edu)

\section{Introduction}

Vortical structures, which are abundant in the ionosphere, manifest themselves in different forms. The auroral spiral, occurring in some auroral arcs, is a prominent vortex structure in optical data (e.g., Davis and Hallinan, 1976; Untiedt et al., 1978; Opgenoorth et al., 1980; Kosch et al., 1998; Lyatsky et al., 2001). The physical dimension of auroral spirals can range between 20 and $1300 \mathrm{~km}$ (Davis and Hallinan, 1976). Some auroral spirals have been associated with auroral breakups (Steen and Collis, 1988), but they can also be observed during the expansion phase, during the recovery phase, and during non-substorm periods (e.g., Partamies et al., 2001a). The development of spirals can vary from a few tens of seconds to minutes (Davis and Hallinan, 1976). Whereas some auroral spirals unwind after formation, others - especially larger ones - do not unwind but instead decay into broken, patchy auroral forms (Davis and Hallinan, 1976). Vortex structures are also identified in equivalent ionospheric currents (EICs) and ionospheric plasma flows (e.g., Untiedt et al., 1978; Küppers et al., 1979), in the absence of auroral spirals (Burke et al., 1983), although some evidence suggests that they are collocated with auroral spirals (Untiedt and Baumjohann, 1993).

Although there are ample ground-based observational descriptions of auroral spirals (e.g., Davis and Hallinan, 1976; Untiedt et al., 1978; Partamies et al., 2001a, 2006), including statistical studies from 216 auroral spirals (Partamies et al., 2001b), their magnetospheric counterparts have been investigated less often. In particular, there are no simultaneous observations of the suspected generator region in the near-Earth plasma sheet, a testimony as to how unlikely it is that a spacecraft traverses magnetic field lines conjugate

Published by Copernicus Publications on behalf of the European Geosciences Union. 


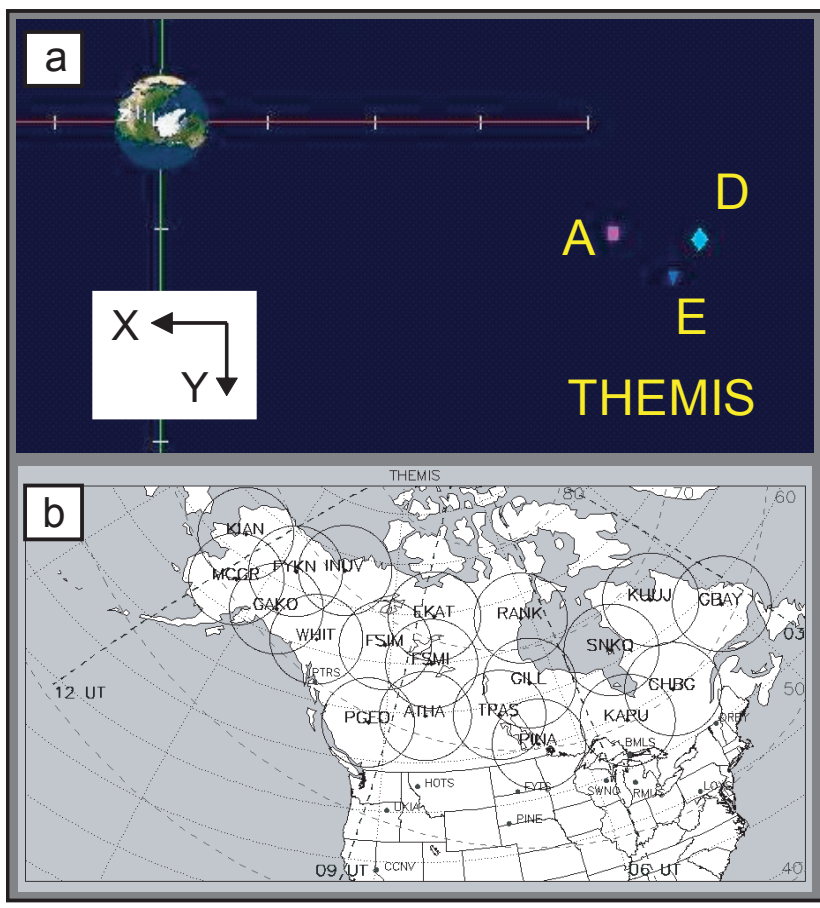

Fig. 1. (a) Locations of the THEMIS spacecraft, projected onto the GSM X-Y plane, during the substorm on 19 February 2008 at 05:25 UT: TH-A (-9.35, 2.26, -2.5), TH-D (-11.17, 2.38, -2.73), and TH-E $(-10.62,3.21,-2.58)$ in GSM coordinates in units of $R_{E}$. The estimated distance from the nominal neutral sheet is less than $0.1 R_{E}$ for all three spacecraft. (b) Locations of the THEMIS ground observatories in Northern America. Circles indicate the fields of view of the all-sky cameras.

to auroral spirals, which have a small size in comparison to the length of an auroral arc. For example, Marklund et al. (1998) reported Freja observations from an altitude of approximately $1700 \mathrm{~km}$ (i.e., outside the generator region) while the spacecraft traversed magnetic field lines adjacent to an auroral spiral and found that the electric field intensified in the direction of the auroral spiral and that the spiral was associated with intense upward field-aligned currents. An association with field-aligned currents has also been observed from ground observations (e.g., Wescott et al., 1975; Untiedt et al., 1978). Some evidence suggests that a line-current like concentration of field-aligned current occurs within auroral spirals (Untiedt and Baumjohann, 1993). The evidence is supported by the model of Hallinan (1976) who attributes spiral formation to a field-aligned current instability.

The auroral spiral remains enigmatic (Haerendel, 2006) since observations in the near-Earth plasma sheet during the formation of auroral spirals do not exist. Therefore, questions remain regarding the magnetospheric driver mechanism for auroral spirals. For example, at the present time, it is not known whether the characteristic spiral shape is formed in the magnetosphere or in the ionosphere. One model has sug- gested that the spiral is the result of a small field line distortion, caused by a current enhancement in the magnetosphere. This distortion grows as it propagates along magnetic field lines towards the ionosphere where it eventually forms a spiral, provided the field line is long enough (Hallinan, 1976). Alternatively, a spiral-like vortex could form in the magnetosphere and map to the ionosphere, where the auroral spiral appears (e.g., Voronkov et al., 2000). In either case, the energy source of the spiral is located in the magnetotail. Therefore, a description of this region during the formation of auroral spirals is needed to ascertain whether a localized current perturbation or a larger-scale magnetospheric instability causes them. Here we provide the first such description. At the end of this report, we compare the observations with the two theoretical models in an attempt to find the generation mechanism for auroral spirals.

\section{Instrumentation}

The observations presented here were obtained from the THEMIS satellites (Angelopoulos, 2008; Sibeck and Angelopoulos, 2008; Bester et al., 2008) and THEMIS groundbased observatories (Donovan et al., 2006; Mende et al., 2008; Russell et al., 2008). Each THEMIS spacecraft carries an identical set of instruments. For this study, we incorporated data from the solid state telescope (SST) (Larson et al., 2009), the electrostatic analyzer (ESA) (McFadden et al., 2008), and the fluxgate magnetometer (FGM) (Auster et al., 2008). Together, both particle detectors (ESA and SST) probed an energy range from a few $\mathrm{eV}$ to $\sim 500 \mathrm{keV}$ for ions at a time resolution of $3 \mathrm{~s}$. The magnetic field vector was sampled at $1 / 3 \mathrm{~Hz}$ (spin resolution) and at $4 \mathrm{~Hz}$. The time resolution of ground magnetometer data was $0.5 \mathrm{~s}$. The allsky imagers (ASIs) had a cadence of $3 \mathrm{~s}$ which allowed us to monitor the auroral spirals in detail.

\section{Observations}

The main focus of our study was to characterize the signatures that accompany the formation of auroral spirals, using both ground-based and spacecraft-based observations. The investigated time period encompassed an isolated substorm on 19 February 2008 during which two auroral spirals occurred, separated by approximately $14 \mathrm{~min}$. At the time of the observations, three THEMIS spacecraft (TH-A, TH-D, and TH-E) were located in the nightside magnetosphere close to the neutral sheet (Fig. 1a). The spacecraft were closely clustered around 23 magnetic local time (MLT) and 9.5 to $12 R_{E}$, with separations in the X-Y (GSM) plane of $\sim 1-2 R_{E}$ while their Z (GSM) separation was less than $0.2 R_{E}$. Locations of the ground observatories and the fields of view of the all-sky cameras are shown in Fig. 1b. The various observations are described in the following subsections. The reader 


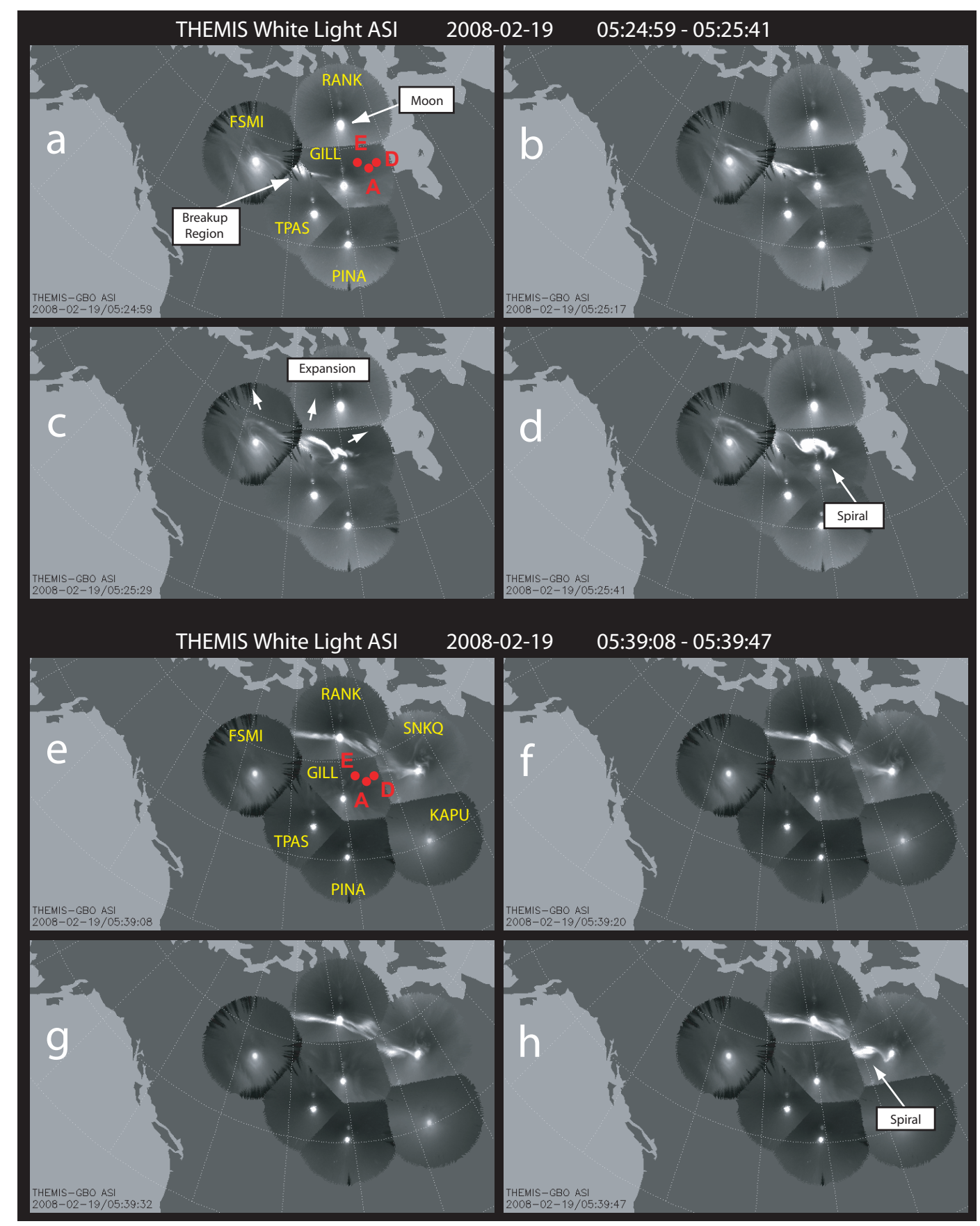

Fig. 2. (a-d) A sequence of ASI images showing the formation of the first auroral spiral on 19 February 2008. Red circles are the footprints of the THEMIS spacecraft as derived from T96. (e-h) A sequence of ASI images showing the formation of the second auroral spiral, approximately 14 min later. The bright spot in each ASI image is the Moon.

is also referred to the study by Keiling et al. (2009) who investigated the generation of the field-aligned current associated with plasma flow vortices in space during this substorm. In our study we will use some of their results.

\subsection{Auroral spirals}

Figure 2a-d shows four ASI images during a 42-s interval. Starting with the auroral breakup (i.e., onset of poleward expansion of the aurora) at $\sim 05: 24: 50$ UT in the field of view of The Pas (TPAS), the aurora rapidly expanded westward and poleward but most intensely eastward. In Fig. 2d, at 


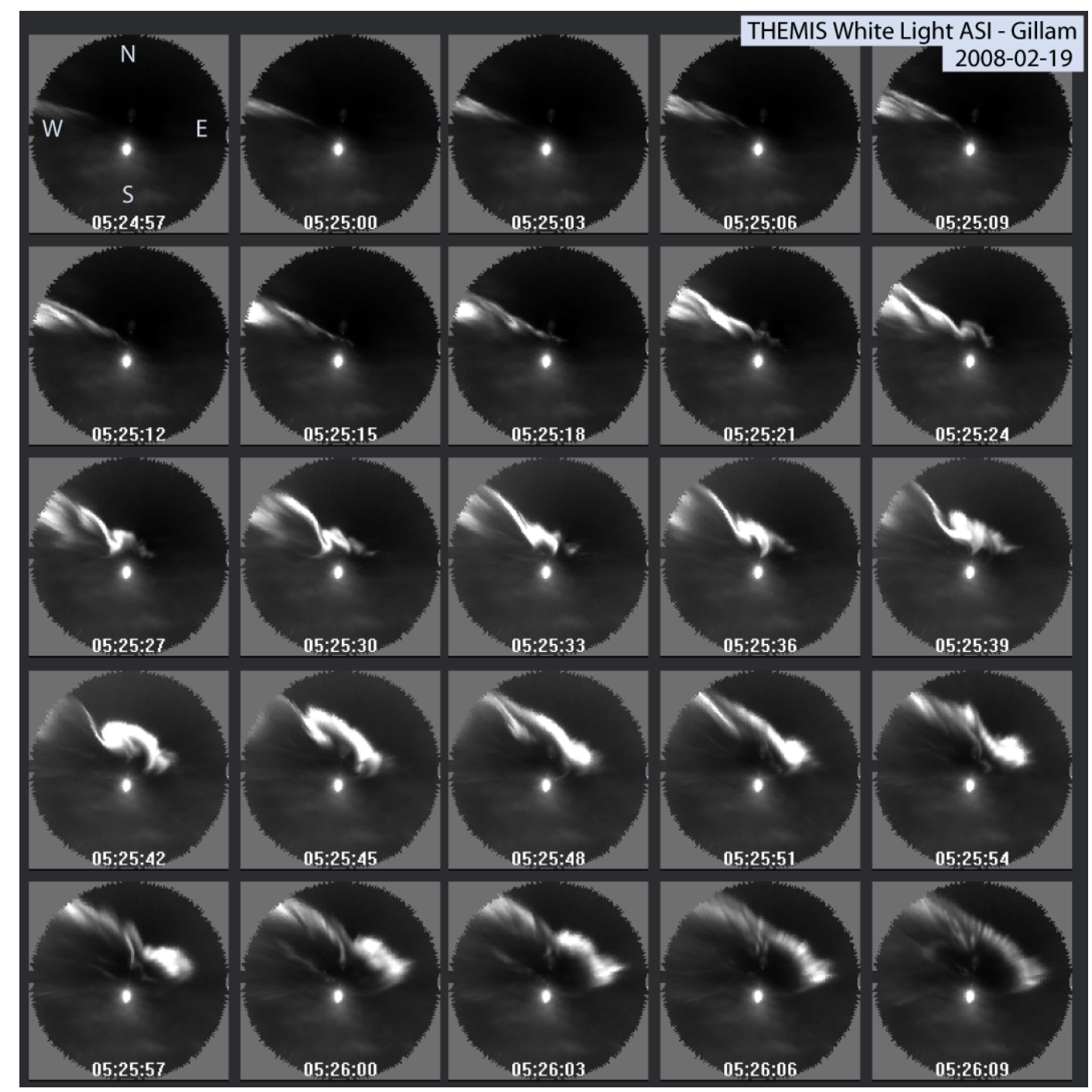

Fig. 3a. Extended sequence of ASI images showing the development of the (a) first and (b) second auroral spiral, recorded at GILL and SNKQ, respectively. Images are three seconds apart. The bright spot in each ASI image is the Moon.

05:25:41 UT, a very prominent auroral spiral (200-300 km in diameter) had formed in the field of view of Gillam (GILL). The second group of ASI images (Fig. 2e-h), starting approximately $14 \mathrm{~min}$ later, covers a time period of $39 \mathrm{~s}$. The aurora had expanded to a higher latitude, and in Fig. 2h, at 05:39:47 UT, a new auroral spiral had formed in the field of view of Sanikiluaq (SNKQ). Between both spiral appearances, the auroral activity subsided and then started at a higher latitude. The red dots in Fig. 2a and e mark the proximate locations for the footprints of the three THEMIS spacecraft, obtained using the T96 magnetospheric model (Tsyganenko, 1995) for mapping. The model's mapping suggests that the spacecraft were not conjugate to the fully developed spirals, at least not to the optical part of the spirals (also see Sect. 3.5).
Figure 3 a consists of a long sequence of consecutive ASI images (3-s cadence) from GILL, showing detail in the development of the first auroral spiral. First, it is noted that the brightening region of the aurora propagated eastward possibly with several arcs (see the image at 05:25:09 UT). The northernmost arc developed undulations, changed into an auroral feature called "fold" (see image at 05:25:30 UT; also see Fig. 12 for enlarged images). Shortly thereafter, at 05:25:42 UT, a counterclockwise (viewed along the magnetic field line) auroral spiral had fully developed, followed by its decay into patchy auroral forms. The time period encompassing the formation, starting with the first undulations within the arc (approximately at 05:25:18 UT), and the decay of the spiral was on the order of one minute, allowing for some ambiguity in determining the beginning and ending of 


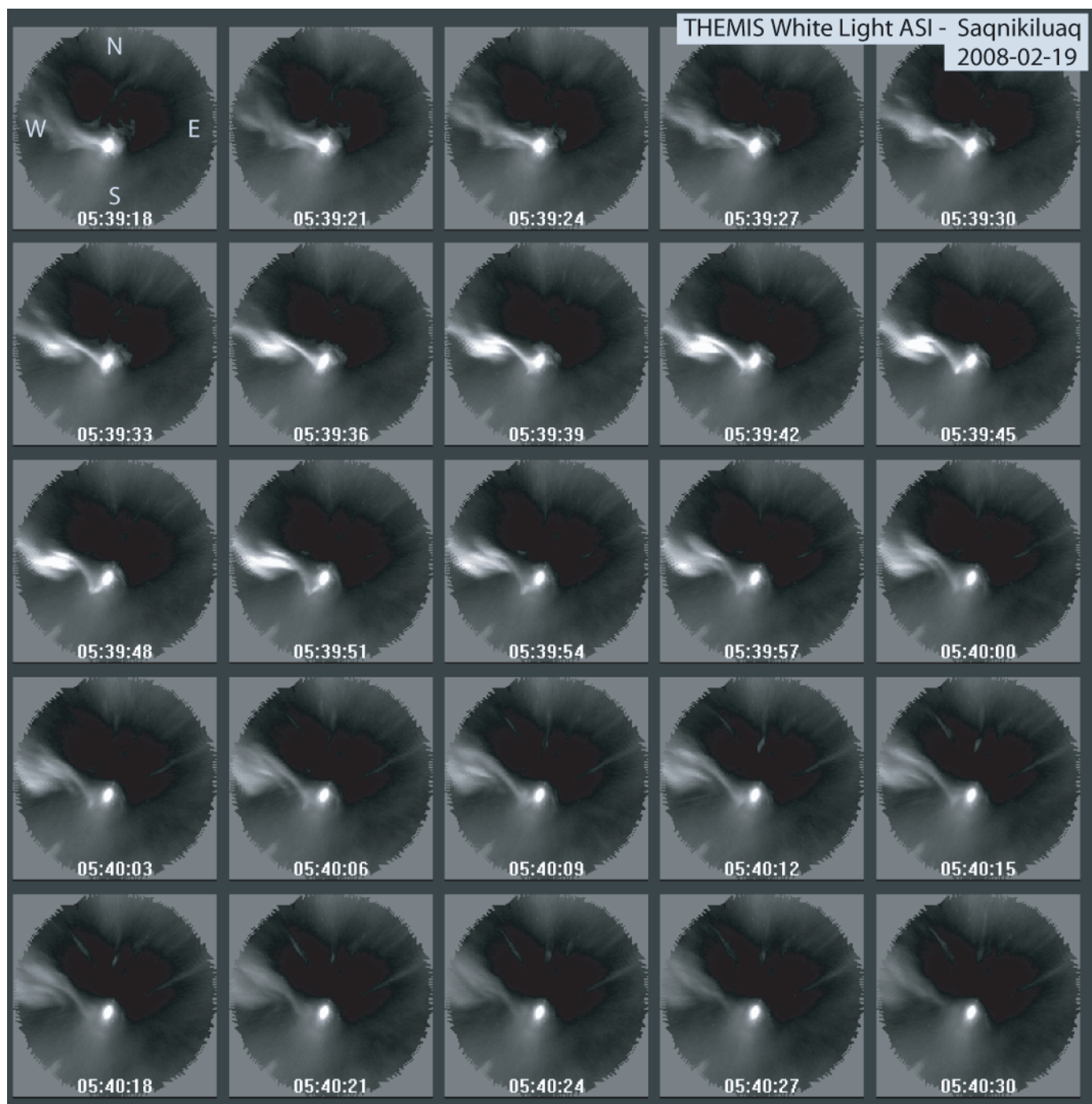

Fig. 3b. Continued.

these processes. The fully-developed spiral was very shortlived, existing for less than $10 \mathrm{~s}$.

Figure $3 \mathrm{~b}$ shows the second spiral in greater detail in the same format as in Fig. 3a. Spiral formation started at approximately 05:39:18 UT, although this time is less certain when compared to the first spiral (Fig. 3a). At 05:39:33 UT, a bright spot formed which appeared to be detached from an arc located northward due to a fainter region in between. The arc continued to bend while the optical intensity of the bright spot and the arc increased, eventually causing them to touch each other. The arc continued to show small changes in its curvature from bent to straight and vice versa, which became successively more difficult to confirm due to a decreasing intensity. Starting at approximately 05:39:54 UT the spiral progressively faded away. Formation and decay of the spiral lasted approximate one minute, similar to the first spiral (Fig. 3a).

\section{2 $H$ bays}

At $\sim 05: 24 \mathrm{UT}$, a negative $H$ bay started to form at Fort Smith (FSMI) and then expanded westward and eastward, as recorded at Fort Simson (FSIM) and Gillam (GILL), respectively (Fig. 4). At $\sim 05: 39$ UT, a second localized $H$ bay formed at higher latitude at Saqnikiluaq (SNKQ) and Kuujjuaq (KUUJ), and a little earlier at Rankin Inlet (RANK) (Fig. 4). The neighboring ground stations (ATHA, TPAS, PINA, KAPU, CHBG) recorded much smaller amplitudes. Remembering that both auroral spirals occurred in the fields of view of GILL and SNKQ at the same time as the $H$ bays formed (data from both stations are shown in the gray panels in Fig. 4), we concluded that each spiral was associated with a localized enhancement of an ionospheric electrojet. 


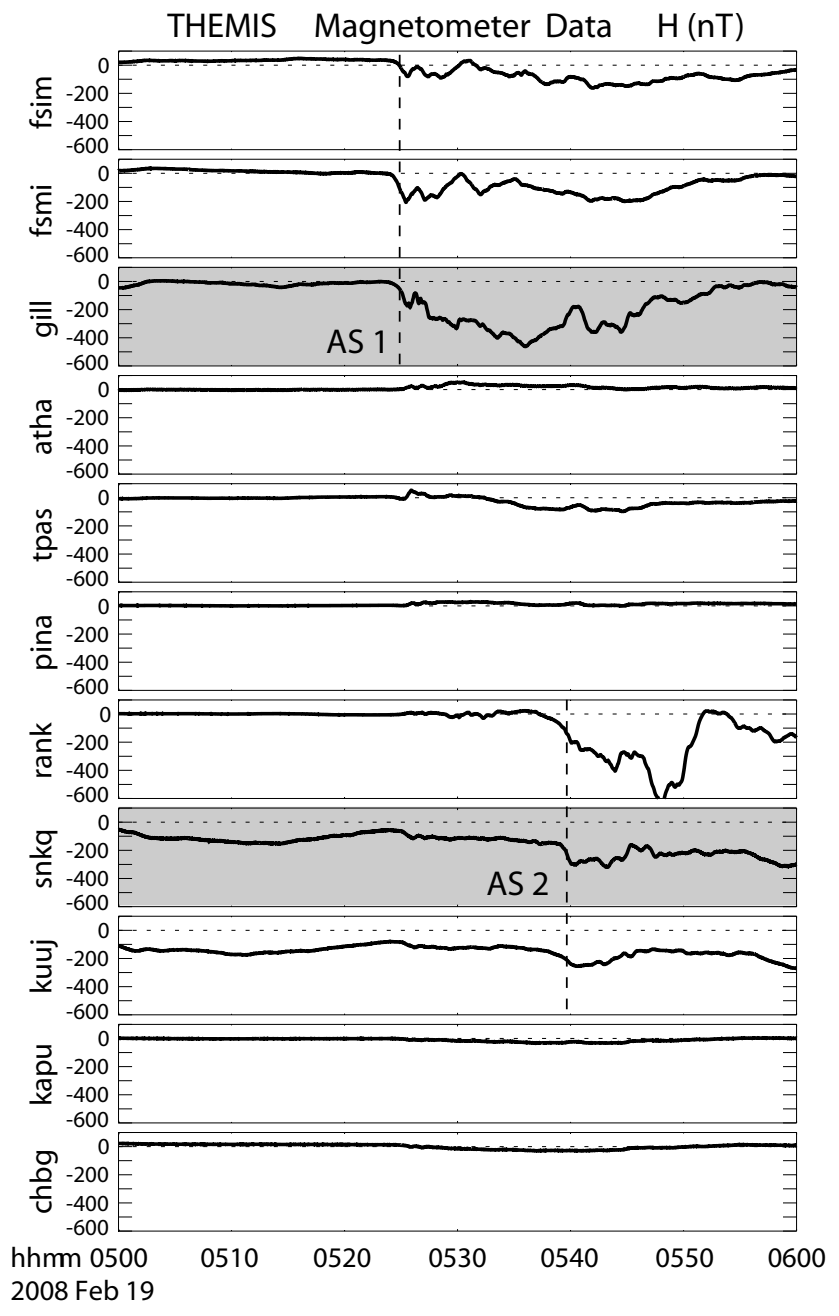

Fig. 4. Ground magnetometer data ( $H$ component) from several THEMIS ground stations on 19 February 2008. Dashed lines mark the times of appearance of the first (AS1) and second (AS2) auroral spirals. The data in the gray shaded panels are from ground stations that recorded the auroral spirals in their ASI images.

\subsection{Equivalent ionospheric currents}

To obtain an estimate for the ionospheric plasma flows and currents, we calculated the equivalent ionospheric currents (EICs) during auroral spiral formations (Fig. 5). EICs were derived with a matrix inversion technique (Amm, 1997; and Amm and Viljanen, 1999) using the measured ground magnetic disturbance of the THEMIS array. The blue vectors indicate the current direction. The opposite direction gives the plasma flow direction, based on the assumption that the ionospheric conductance was uniform. Before the formation of the first auroral spiral (panel a), the EICs were relatively weak with an identifiable eastward electrojet. After 05:24 UT, the westward EIC electrojet intensified (panel b). Oppositely rotating EIC vortices started to form at approximately 05:24:10 UT (not shown) by showing first signs of changing the directions of the EICs and were developed by 05:24:40 UT (panels $b$ and c). The dot and the cross show the respective center of the vortices. Clockwise and counterclockwise rotations when viewed along the magnetic field direction are associated with downward and upward fieldaligned currents (FACs), respectively (e.g., Untiedt et al., 1978; Opgenoorth et al., 1980). The poleward rim of the upward-current EIC vortex coincided with the breakup region of the brightening aurora (panel b). Immediately after auroral breakup, both the auroral brightening region (cf. Fig. 2a-c) and the upward-current EIC vortex moved eastward (see the dots in panels $b$ and $c$ ) while intensifying. The auroral spiral, reported in Sect. 3.1, formed in the transition region between both vortices, a region of enhanced EICs (panel c). It is also noted that the expanding aurora had a shape similar to that of the intense EICs (arrows with $>200 \mathrm{~mA} / \mathrm{m}$ ) and occurred at approximately the same location.

At the time of the second auroral spiral, approximately 14 min later, the EIC pattern had significantly shifted towards the north (Fig. 5b). Clockwise and counterclockwise EIC vortices can still be identified with the center of the clockwise EIC vortex at a higher latitude as compared to its location during the first auroral spiral. Additionally, the optical signature had changed significantly with auroral arcs at higher latitudes as well. As for the first auroral spiral, the shape of the most intense EICs was similar to the shape of the auroral arcs. At approximately 05:40 UT (panel f), the EICs had undergone another significant change, namely a northward widening. The lower edge of this region approximately coincides with the location of the auroral spiral (see arrows labeled "spiral").

\subsection{Riometer}

The eastward and poleward propagation of the substorm disturbance as seen in the ground magnetometer data and the optical data can also be seen as absorption bays in the riometer data (Fig. 6). Such bays have been shown to be associated with particle injections in the conjugate near-Earth plasma sheet (Spanswick et al., 2007). In Fig. 6, the stations are arranged from top to bottom according to the E-W Line and the Churchill Line. Dashed red lines emphasize the time delays of the bays along each line. The data also suggest that the first auroral spiral (AS1), as seen in the field of view of GILL, was conjugate to particle injections in space (see the absorption bay at GILL), which is supported by direct space observations as shown in Sect. 3.5. On the other hand, for the second spiral (AS2), which occurred in the field of view of SNKQ, it cannot be verified from riometer data that it was conjugate to particle injections because no riometer station was located at SNKQ or nearby. However, as seen in the next section, a particle injection was indeed recorded in space during the second spiral as well. 


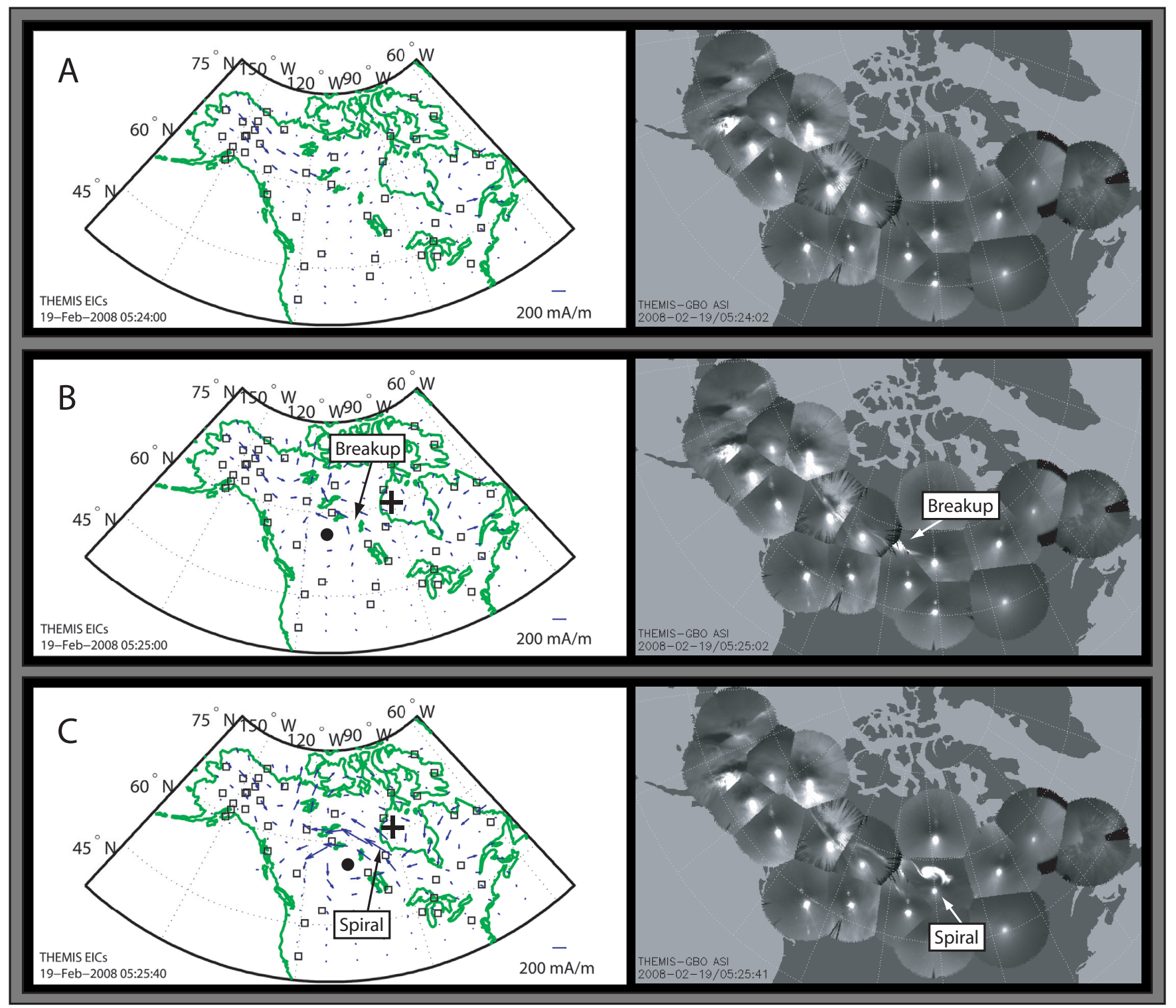

Fig. 5a. Equivalent ionospheric currents (EICs) in comparison to ASI images during the (a) first and (b) second auroral spiral on 19 February 2008. The dot and cross, overlaid on the EICs, mark the center of counter-clockwise and clockwise EIC vortices. The open squares mark the locations of the ground stations used for the EIC calculations.

\subsection{Space observations}

Based on field line mapping (see Sect. 3.1), the THEMIS spacecraft were not conjugate (but near) to the visible parts of the fully developed auroral spirals. However, the reader is cautioned that field line mapping is uncertain and controversial, particularly during times of substorms which prevailed during this event. The spacecraft data are nevertheless of interest because the region of the magnetospheric counterpart of the spiral is not only limited to the region conjugate to the optical part and because of a paucity of reports showing a comparable constellation between ground-based and spacebased observations during the formation of auroral spirals.
Concurrent with both auroral spirals (see labels AS1 and AS2 in Fig. 7), plasma flow and magnetic field data revealed that two larger disturbances occurred: dipolarizations and enhanced plasma flows. The dipolarization signatures in the three magnetic field components differed for the various spacecraft and during both auroral spirals, which can be explained by the closeness of the spacecraft to the neutral sheet ( $<0.2 R_{E}$ to the nominal neutral sheet) and the sudden appearance of flow vortices. Under these conditions, the flow vortices "sloshed" the magnetic field along in the high-beta plasma, and, as a result, the orientation of the magnetic field deviated significantly from the orientation before the appearance of the flow vortices. For example, the strong 


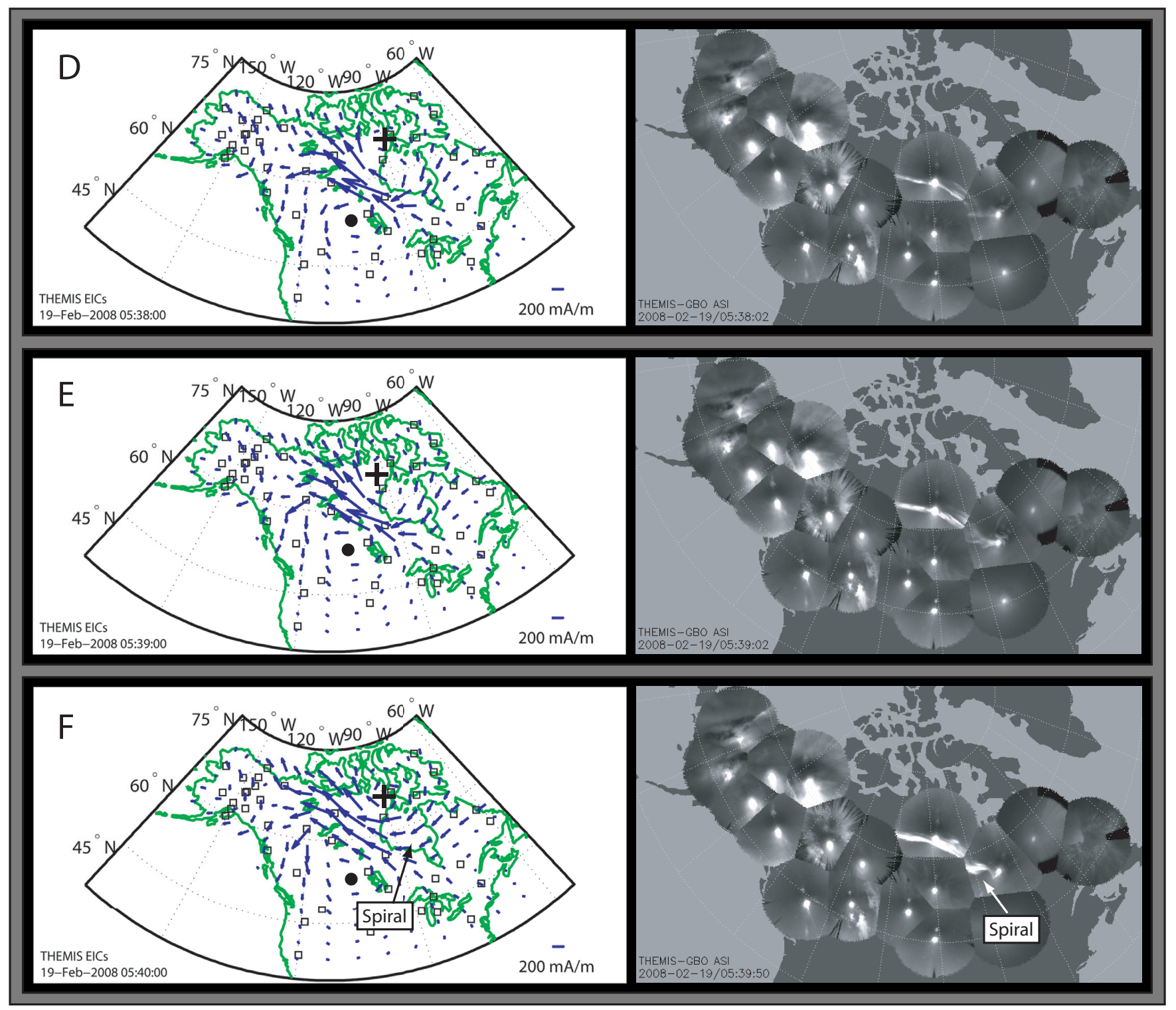

Fig. 5b. Continued.

$B_{y}$ deviation at TH-E (Fig. 7b) during AS2 provided most of the total magnetic field, whereas before AS2 it was the $B_{z}$ component. However, a calculation of the total magnetic field (not shown) confirms the occurrence of dipolarizations.

The enhanced plasma flows during both spirals (AS1 and AS2) (Fig. 7d-f) can be identified as vortical flows which can best be seen when plotted as projections of the velocity vectors in the X-Y (GSM) plane (Fig. 7g-i). At approximately 05:24:00 UT and 05:38:05 UT (for the first and second vortical structure, respectively), the clustered spacecraft (TH-A, D, and E) registered the first signs of small changes in the flow direction, followed by more rapid flow enhancements towards and/or away from Earth $\left(\left|V_{x}\right|>0\right)$ at
05:25:00 UT and 05:39:10 UT. The initial changes could be interpreted as the pre-existing plasma being pushed out of the way by the arriving flows of the vortex. We note that Keiling et al. (2009) reported the onset (enhanced $V_{x}>0$ ) of a separate flow vortex, located two hours (MLT) to the west of the three clustered THEMIS spacecraft, at approximately 05:23:55 UT, using a fourth THEMIS spacecraft (THC). It was argued that this additional vortex was the counterrotating flow vortex that was driving the counterclockwise EIC vortex in the ionosphere (see Sect. 3.3). We note that an inspection of data (not shown) from $\mathrm{TH}-\mathrm{C}$ during the second vortex (near 05:38 UT) did not show signs of a counterrotating vortex. We believe that this is simply due to the 


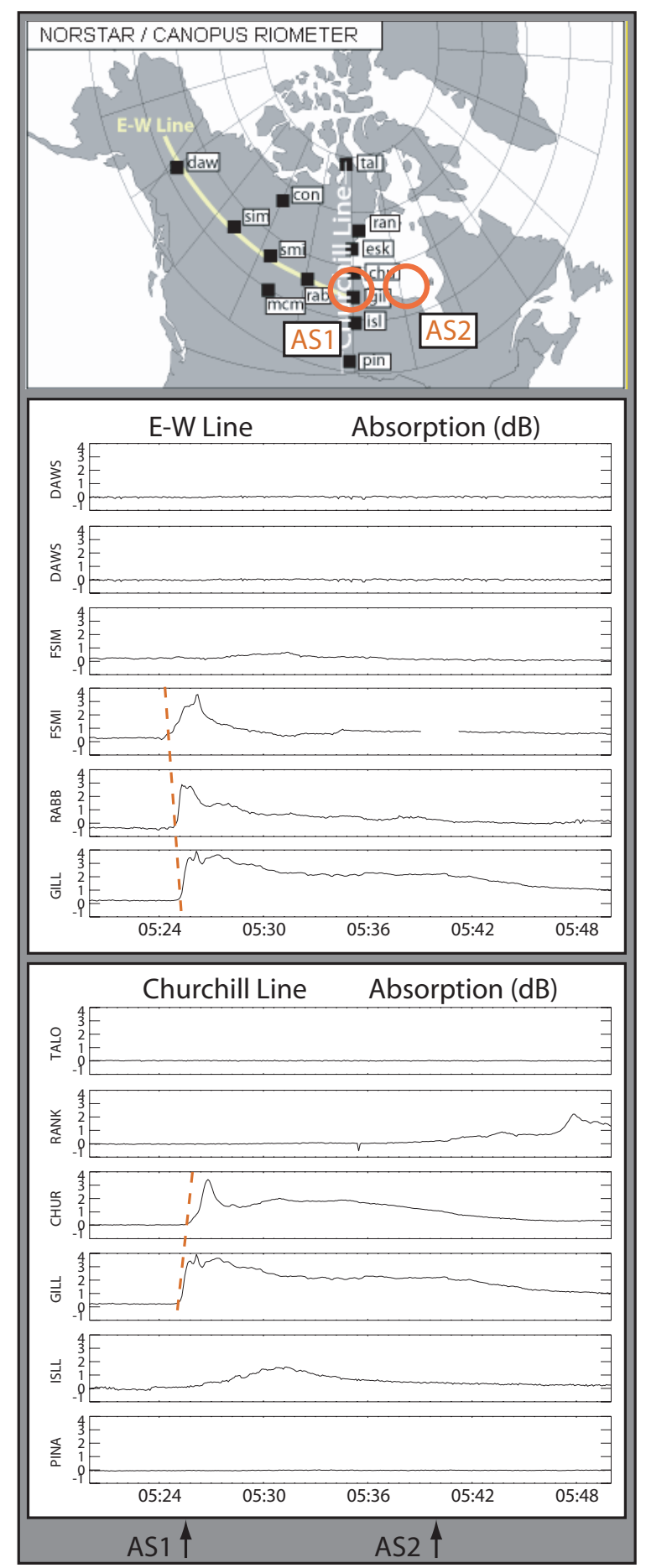

Fig. 6. Riometer data on 19 February 2008 ordered along two lines: the E-W line and the Churchill line. Red circles show the locations of the first (AS1) and second (AS2) auroral spiral. The appearance times for both auroral spirals are indicated at the bottom of the figure by arrows, labeled AS1 and AS2. The red dashed lines emphasize the time delay of the formation of absorption bays.

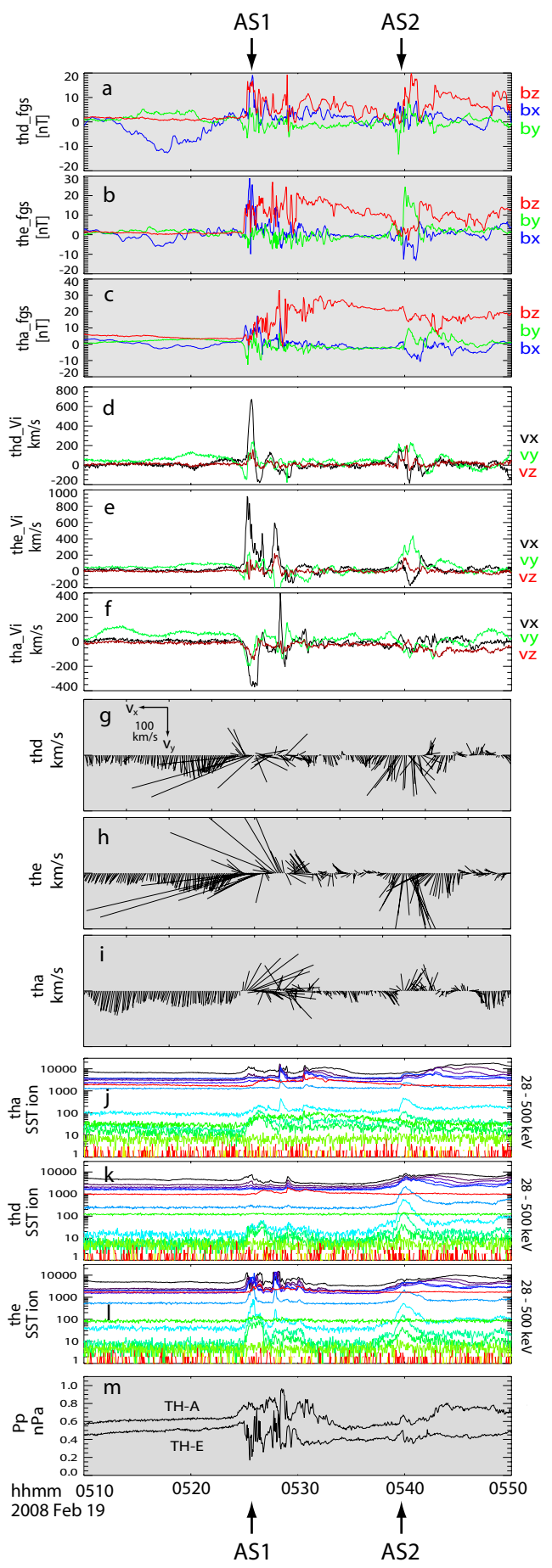

Fig. 7. Spacecraft data from three THEMIS probes (TH-D, TH-E, and TH-A, in this order) on 19 February 2008: (a-c) magnetic field; (d-f) plasma flow velocities calculated using data from both ESA and SST instruments; $(\mathbf{g}-\mathbf{i})$ vector plots of flow velocities projected onto the X-Y GSM plane; (j-l) differential ion energy flux for various energy channels from the SST instrument in the range from $25 \mathrm{keV}$ to $\sim 500 \mathrm{keV}$; and $(\mathrm{m})$ particle pressure derived from both ESA and SST data. 
fact that this vortex occurred at a different location and was therefore simply missed by TH-C (see Fig. 5 which shows that the corresponding EIC vortex had indeed moved further east and south). Each vortex lasted for approximately 1-2 min. In comparison, the auroral spirals started to develop at 05:25:18 UT and 05:39:18 UT (i.e., more than one minute after the first sign of vortex activity in space) and took about $20-30$ s to completely form (cf. Sect. 3.1). We also mention that Keiling et al. (2009) estimated that the first vortical structure generated currents along the magnetic field into and away from the downward-current EIC vortex of approximately $2.8 \mathrm{nA} \mathrm{m}^{-2}\left(14 \mu \mathrm{A} \mathrm{m}^{-2}\right.$ mapped to the ionospheric altitude) or a total current of $\sim 0.1 \mathrm{MA}$.

Furthermore, ion injections were recorded at the times of the spiral developments (see labels AS1 and AS2 in Fig. 7). Figure 7j-1 shows differential ion energy fluxes for several energy channels covering the range from $25 \mathrm{keV}$ to $\sim 500 \mathrm{keV}$. The injections were nearly dispersionless, suggesting that the source was close to the locations of the spacecraft.

Particle pressures (calculated from both ESA and SST data) show significant changes at the times of the spiral formations (Fig. 7m). TH-A and TH-E, which were radially separated gave evidence for a pressure gradient towards Earth of approximately $0.15 \mathrm{nPa} / R_{E}$ before the first auroral spiral (AS1) and of approximately $0.4 \mathrm{nPa} / R_{E}$ during the spiral. The second spiral (AS2) showed smaller pressure changes, possibly because the spacecraft was farther away from the field lines that were conjugate to the optical spiral.

Returning to the velocity vector projections (Fig. $7 \mathrm{~g}-\mathrm{i}$ ), we estimate the velocity shear associated with the plasma flow vortices. The flow contained inside the first vortex (at $\sim 05: 24$ UT) was directed in opposite directions, yielding velocity differences from edge-to-edge of up to $1000 \mathrm{~km} / \mathrm{s}$. Because of the nearly circular flow pattern at times, as verified by the clustered spacecraft which were separated by approximately $1 R_{E}$ (Fig. 6 in Keiling et al., 2009), we apply $1 R_{E}$ as a scale size for the spatial gradient of the velocity shear. Therefore, we estimate the peak velocity shear to be on the order of $1000 \mathrm{~km} /\left(\mathrm{s} R_{E}\right)$. In addition, a shear flow existed between the centers of the two counter-rotating vortices, whose ionospheric signatures were the two EIC vortices (Fig. 5a). This shear flow is unidirectional, being near zero at each vortex center and reaching peak values of at least $800 \mathrm{~km} / \mathrm{s}$ (e.g., at TH-E) between both centers. The associated velocity shear is less certain since no reliable estimate for the separation of the two centers is available from space measurements. The flow velocities associated with the second vortex in space (at $\sim 05: 39 \mathrm{UT})$ are smaller $(<500 \mathrm{~km} / \mathrm{s})$, and therefore the velocity shear is likely smaller than during the first vortex.

\section{Summary and discussion}

Here we reported simultaneous observations of auroral spirals and their generation region in the near-Earth plasma sheet, using the THEMIS spacecraft and the THEMIS ground network of all-sky imagers and magnetometers. The observations were made during a substorm on 19 February 2008 which showed two consecutive large-scale (200$300 \mathrm{~km}$ in diameter) auroral spirals separated by $\sim 14 \mathrm{~min}$. The spirals formed during auroral expansion and subsequent intensification. Formation and decay together lasted on the order of one minute for each spiral. Both the spirals were associated with vortical plasma flows, dipolarization and particle injection in space. The aurora reached a nearly quiet phase between both spirals.

Concurrent with both auroral spirals, a pair of EIC vortices with opposite rotations (corresponding to upward and downward currents) existed in the ionosphere. The region between the centers of both EIC vortices showed enhanced EIC currents. The brightened aurora and the spirals approximately coincided with these enhanced current regions (cf. Fig. 8a). Intense EICs can correspond to intense horizontal ionospheric plasma flows if the ionospheric conductance is uniform. Although uniform conductance is typically not expected, in some cases a good correspondence between EICs and horizontal flow, as determined from radar measurements, has been shown (Weygand et al., 2009; Kivelson et al., 2009). Thus, the region between both EIC vortices might indeed have been a region of strong plasma flow. In fact, an association between strong shear flows and auroral spirals has previously been proposed (e.g., Steen and Collis, 1988).

Some differences in the evolution of the EIC vortices existed during both auroral spirals. During the first spiral, the upward-current EIC vortex moved eastward after auroral breakup while intensifying; similarly, the most intense aurora expanded predominantly eastward. During the second spiral formation, the intense EIC currents abruptly jumped poleward and broadened. In both cases, the spirals formed in the transition region between clockwise and counterclockwise EIC vortices, a region of intense EICs (cf. Fig. 8a). The transition region also shows variations in the current intensity (identifiable in the changing lengths of the arrows) from one vortex center to the other. Such current intensity gradients (i.e., curl of EIC) are associated with FACs, given the right ionospheric conductance (Amm and Viljanen, 1999). The similar shape of the region of intense EICs and the bright aurora is also evidence that these EIC regions are connected to FACs, since auroral arcs and spirals are regions of strong upward currents (e.g., Wescott et al., 1975; Untiedt et al., 1978; Marklund et al., 1998). The reader might want to compare our observations with those from Untiedt and Baumjohann (1993) who reported on auroral spirals centered on an EIC vortex.

In addition to an upward current sheet above the auroral arc, it is expected that a downward current sheet is located 


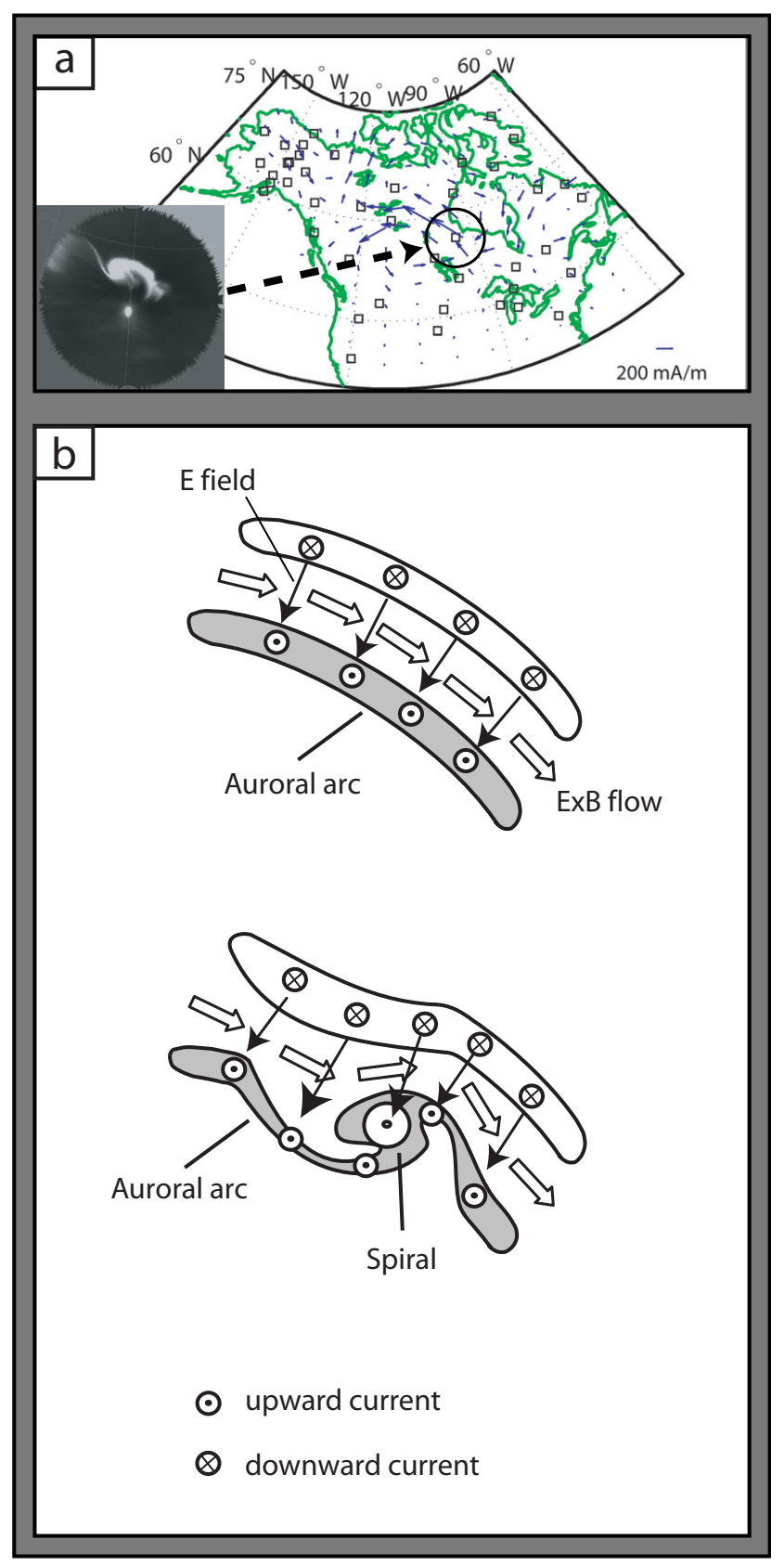

Fig. 8. Relationship among EICs, $\boldsymbol{E} \times \boldsymbol{B}$ flows, upward and downward currents, and auroral spirals. (Top) The auroral spiral (see inset) is collocated with intense EICs, which also correspond to enhanced $\boldsymbol{E} \times \boldsymbol{B}$ flows in the ionosphere. (Bottom) Schematic of several physical quantities in the ionosphere before and at auroral spiral formation.

either poleward or equatorward of the arc, depending on whether the arc is located in the westward or eastward electrojet region, respectively (e.g., Aikio et al., 1993). Figure $8 \mathrm{~b}$ (top) schematically shows such downward and upward FAC sheets, which are closed via ionospheric Pederson currents, before the auroral spiral formation on 19 February
2008. Upward and downward currents approximately correspond to the two oppositely rotating EIC vortices (Fig. 8a), while allowing for the possibility that the outer parts (and not the center) of the vortices correspond to the current sheets (e.g., Kosch et al., 2000). The $\boldsymbol{E} \times \boldsymbol{B}$ drift of ionospheric plasma and the direction of the electric field, as illustrated in Fig. 8b, were inferred from the EICs (Fig. 8a), assuming uniform conductance. In this case, the EICs equal the Hall currents which flow in the $-(\boldsymbol{E} \times \boldsymbol{B})$ direction. Hence, the opposite of the EIC direction yields an estimate of the convectional flow direction. Note that the stations' separations in the magnetometer network dictate the spatial resolution of the EIC calculations, which is not high enough to resolve smaller features - such as the auroral spirals - in the EICs.

When the auroral spiral formed (Fig. 8b, bottom) the intensity of the upward FAC increased as indirectly inferred from the increasing brightness of the aurora (cf. Fig. 8a). Evidence for enhanced currents also came from localized $H$ bays (which were also included in the calculations of the EICs), occurring at the stations that recorded the auroral spiral. A correlation between $H$ bays and aurora spirals was reported by Wescott et al. (1975). Furthermore, for this substorm (19 February 2008) the longitudinal locations of upward and downward FACs, as well as their magnitude as a function of time were modeled by Keiling et al. (2009) to fit the midlatitude magnetic field variations (Fig. 9). According to the model, the FACs started to develop before 05:25 UT with upward and downward currents located at $\sim 20.5$ MLT and $\sim 23$ MLT, respectively. The meridian of the downward current coincided with those of the footprints of TH-A, TH-D and TH-E (labels a, d, and e). The current magnitude gradually increased and reached a peak value of $\sim 0.7$ MA. It was concluded by Keiling et al. (2009) that the forming plasma vortex in space accounted for $\sim 0.1 \mathrm{MA}$ during the initial phase of the substorm, but that at later times other current mechanisms took over. This initial vortexrelated current increase coincided with the time of the first auroral spiral (AS1). During the period of decreasing current after 05:35 UT, the current showed an upward deviation (line above the dashed line) from the general decaying trend (dashed line) at around 05:40 UT which accounted for approximately $0.1 \mathrm{MA}$. This time coincided with the formation of the second auroral spiral (AS2). Therefore, it is possible that the concurrent second plasma vortex in space created this current enhancement which was superposed over the other, stronger current created by other mechanisms and which is similar in magnitude to the current enhancement associated with the first spiral (AS1). Also noted is that the longitude of the downward FAC jumped by approximately $0.4 \mathrm{~h}$ MLT to the east during both auroral spirals. These jumps could be caused by the effect on the magnetic mapping (Donovan, 1993) of the spiral-associated current enhancements. We conclude that both auroral spirals were likely associated with current enhancements generated in space by the flow vortices. 


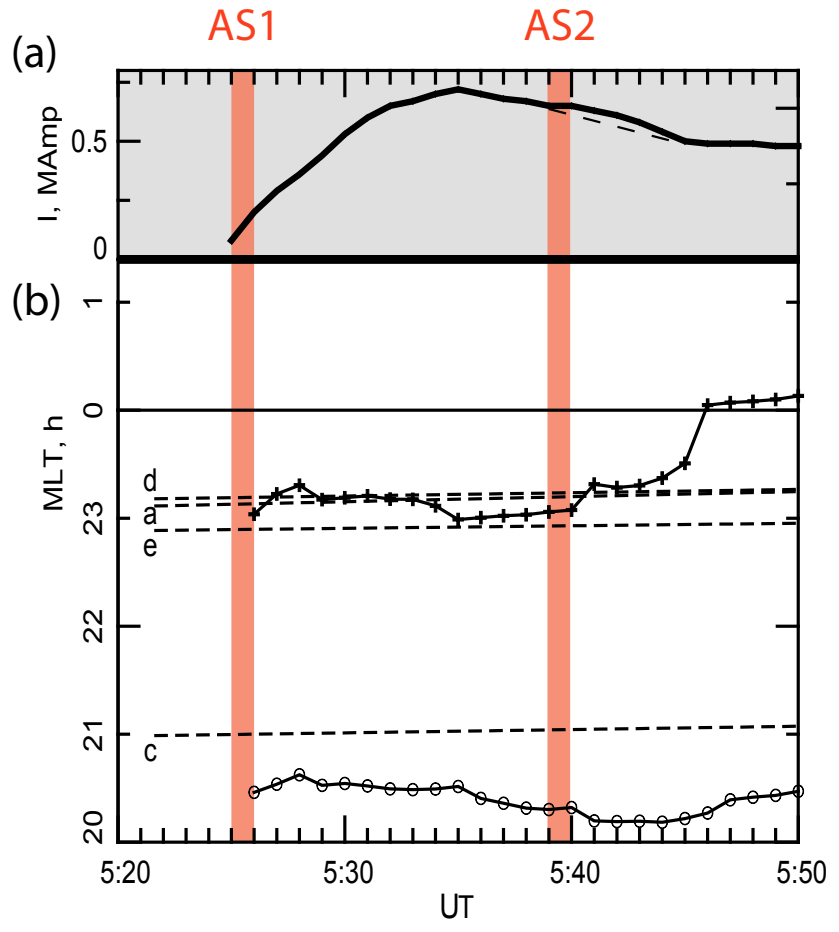

Fig. 9. Reconstructed substorm current wedge (SCW) using THEMIS and INTERMAGNET ground magnetometer data. The longitudinal locations of upward and downward FACs as well as their magnitude as a function of time were modeled to fit the midlatitude magnetic variations. The vertical red bars mark 5-min intervals during which both auroral spirals occurred. (a) Current intensity of the SCW (note that the dashed line follows the earlier trend and is not modeled data). (b) Longitude in MLT of downward FAC (crosses), upward FAC (circles), and footprints of TH-A, TH$\mathrm{C}$, TH-D, and TH-E (dashed lines) (modified from Keiling et al., 2009).

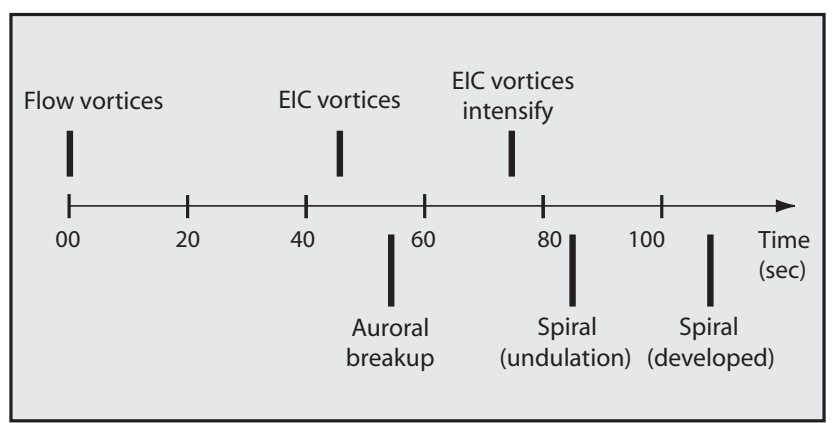

Fig. 10. Sequence of events for the first auroral spiral on 19 February 2008. Times are displayed relative to the first sign of a flow vortex in space (i.e., at TH-C) and can be found in Table 1. See the text for further description.

Taking the onsets of different physical phenomena (summarized in Table 1) into account, one can construct the following scenario for the first auroral spiral formation. A
Table 1. Summary of various onset times (approximate): the auroral breakup, the auroral spirals as recorded in the ASI data, the EIC vortices as determined from ground magnetometer data, and the vortical plasma flows in space as recorded by the THEMIS spacecraft.

\begin{tabular}{lcc}
\hline Phenomenon & First event (UT) & Second event (UT) \\
\hline Auroral breakup & $05: 24: 50^{\mathrm{a}}$ & N/A \\
Flow vortices in space & $05: 23: 55^{\mathrm{b}}$ & $05: 38: 05^{\mathrm{c}}$ \\
& $05: 24: 00^{\mathrm{c}}$ & $05: 39: 10^{\mathrm{d}}$ \\
& $05: 25: 00^{\mathrm{d}}$ & \\
& $05: 24: 10^{\mathrm{e}}$ & continuation \\
EIC vortices & $05: 24: 40^{\mathrm{f}}$ & \\
& $05: 25: 10^{\mathrm{g}}$ & \\
& $05: 25: 00$ & $05: 38: 55$ \\
Ion injections & $05: 25: 18^{\mathrm{h}}$ & $05: 39: 18^{\mathrm{h}}$ \\
Auroral spiral & $05: 25: 42^{\mathrm{i}}$ & $05: 39: 45^{\mathrm{i}}$ \\
\hline
\end{tabular}

a onset of poleward expansion of aurora

$\mathrm{b}$ inward motion of plasma $\left(V_{x}>0\right)$ at TH-C

${ }^{\mathrm{c}}$ begin of a rotation of the velocity vector at the clustered spacecraft

$\mathrm{d}$ inward and/or outward motion of plasma $\left(\left|V_{x}\right|>0\right)$ at the clustered spacecraft

e begin of a rotation of EICs

${ }^{\mathrm{f}}$ EIC vortices are formed

$\mathrm{g}$ intensification of EICs

$\mathrm{h}$ undulation in auroral arc

i fully developed auroral spiral

partial sequence of events is also graphically presented in Fig. 10. The times are displayed relative to the first sign of a flow vortex in space (at TH-C). A pair of plasma flow vortices formed in the near-Earth plasma sheet and were first recorded by TH-C at $\sim 05: 23: 55$ UT, followed by the three clustered spacecraft (TH-A, TH-D, and TH-E). At 05:24:40 UT, EIC vortices had formed in the ionosphere and continued to intensify. These EIC vortices were initiated by the plasma flow vortices that generated currents along the magnetic field into the EIC vortices. In the region between the two EIC vortex centers, intense EICs were collocated with an auroral arc which started to undulate at approximately 05:25:18 UT, i.e., $38 \mathrm{~s}$ after the EIC vortices had formed. This time also approximately coincided with an intensification of the EICs at $\sim 05: 25: 10$ UT. Interestingly, the upward-current EIC vortex was moving eastward towards the downward-current EIC vortex, and approximately when their centers were closest, formation of the auroral spiral began. At approximately 05:25:42 UT (i.e., $107 \mathrm{~s}$ after the first plasma vortex signature in space), the spiral was fully developed. The vortex-driven current that powered the spiral continued to grow, causing the saturation of the auroral spiral (i.e., a breakup into patchy 


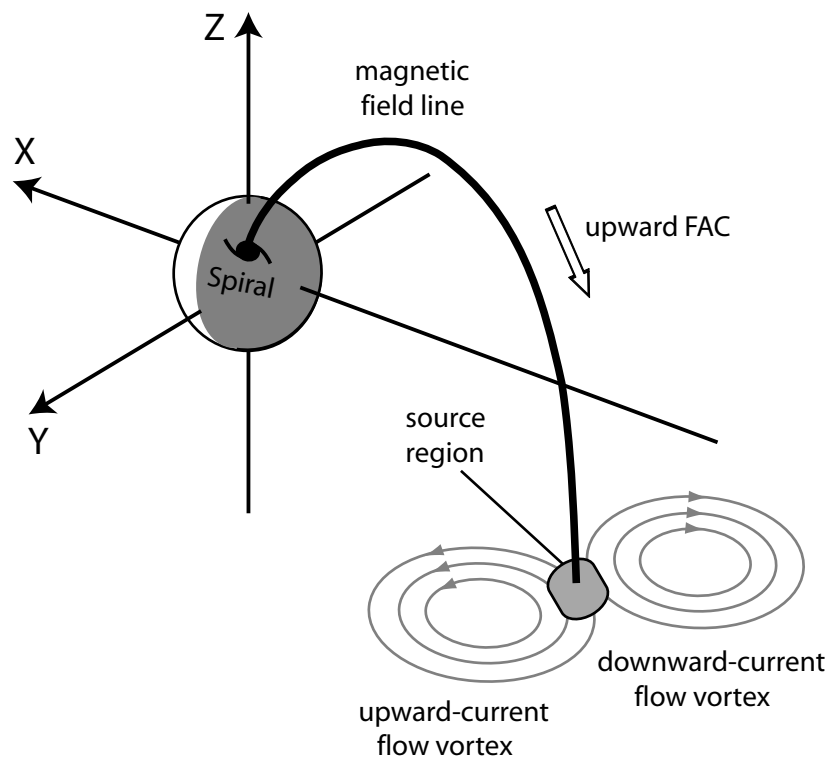

Fig. 11. Illustration of the connection of an auroral spiral and its source region in the magnetosphere, located between two oppositely rotating plasma vortices. The circular shape of the vortices is idealized and is likely more stretched and irregular in reality. The source region maps into an ionospheric region of curl of EIC, indicating the existence of field-aligned currents, where the auroral spiral occurs.

auroral forms). A local electrojet enhancement was also related to the formation of the second spiral. Similar to the first spiral, the current enhancement was caused by a plasma flow vortex. Overall, the signatures were not as strong as for the first auroral spiral, which could have been the reason why the second spiral was not as intense and did not saturate but simply faded away.

For clarification, we emphasize that in the scenario described above the optical spiral was not the ionospheric footprint of the magnetospheric plasma flow vortex; rather, it was the result of some process that occurred in the transition region between the vortex centers where strong shear flows existed (i.e., the edges of the flow vortices), as illustrated in Fig. 11. However, the flow vortices were essential for providing the energy and the necessary conditions for the spiral generation process. We now discuss two models that have the potential of providing this process. Early on, Hallinan (1976) provided a theoretical explanation for the formation of spirals. In his model a spiral is the result of an upward current enhancement within an extended current sheet, if the current intensity is larger than $2.5 \mu \mathrm{A} \mathrm{m}^{-2}$. The model describes spatial variations along the magnetic field line from the magnetospheric end to the ionospheric end (top of Fig. 12) rather than the temporal development of a spiral. The temporal development for our first auroral spiral event (19 February 2008), as captured in the ASI images (bottom of Fig. 12), is remarkably similar to the spatial variations of Hallinan's model. Furthermore, the current density associ-

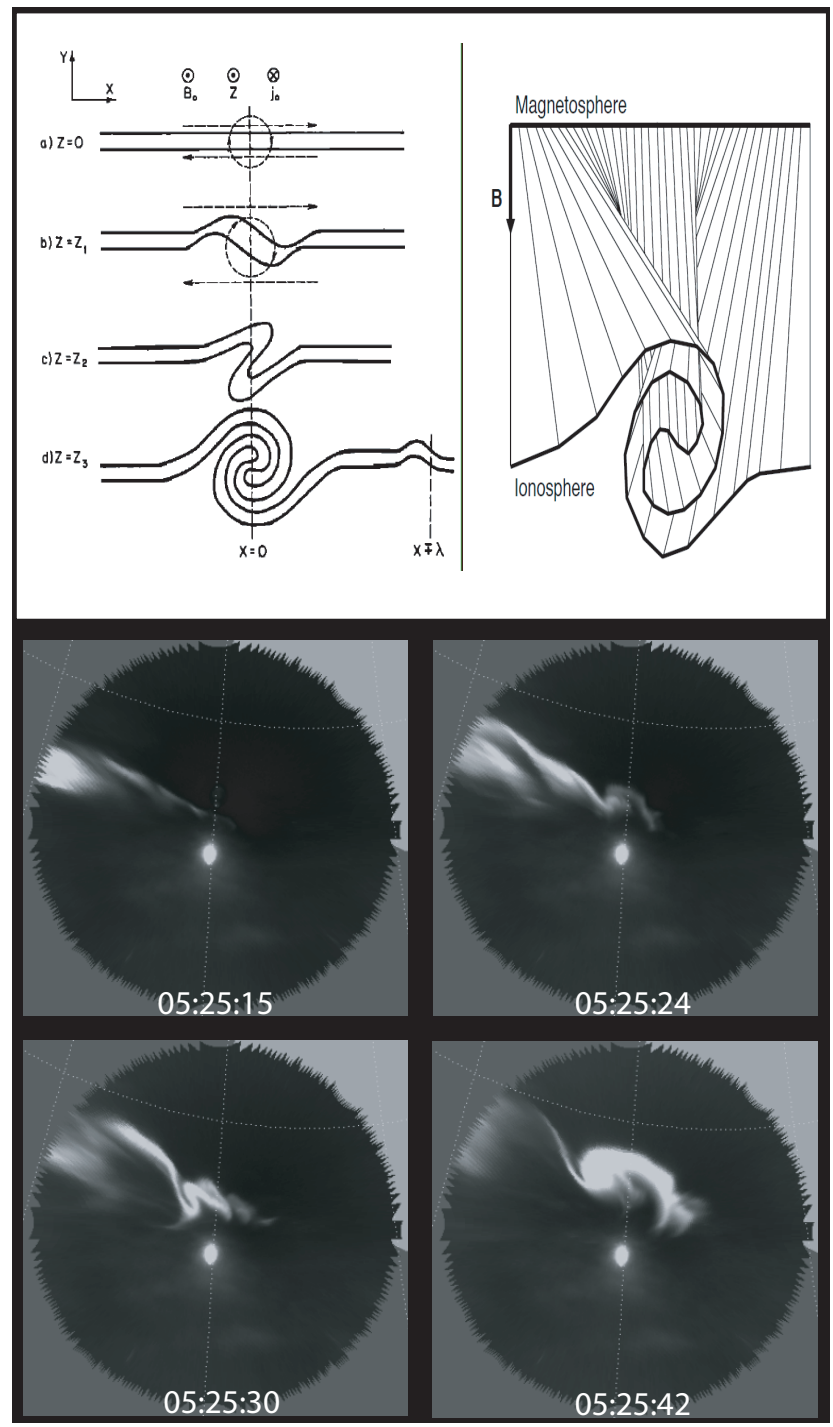

Fig. 12. Top: Illustration of field line deformation from the magnetosphere to the ionosphere in association with auroral spirals. The left figure is from Hallinan (1976). The right figure is from Partamies (2004). Bottom: Selected ASI images showing different stages during the auroral spiral formation on 19 February 2008.

ated with the downward EIC vortex was $\sim 14 \mu \mathrm{A} \mathrm{m}^{-2}$ at the top of the ionosphere. If we assume that the upward current, which was fed by the downward current, had a similar density, then the criterion for spiral formation - according to Hallinan (1976) - was satisfied. It is even more likely that the upward current density was larger because the region of upward current is typically more confined spatially (e.g., Untiedt and Baumjohann, 1993). In the model, the source of the current enhancement is however not addressed. Our observations suggest that the current source for auroral spirals can be plasma flow vortices in the magnetosphere. Finally, we note that a spiral saturation which was observed for the first auroral spiral has not been addressed in the model either. 


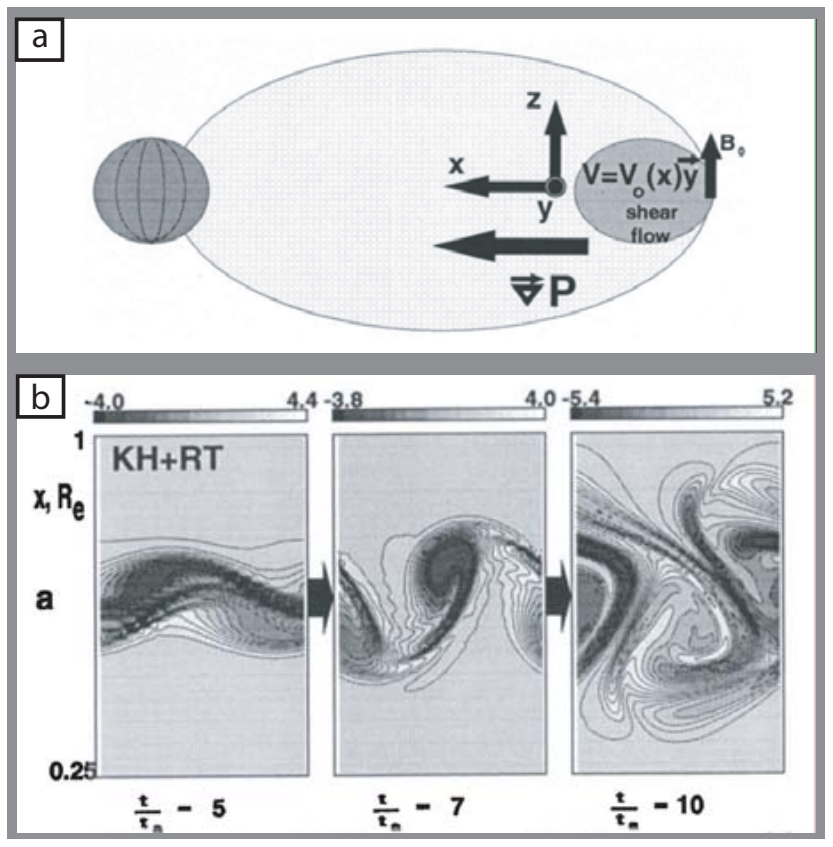

Fig. 13. (a) Illustration from Voronkov et al. (1997), showing a shear flow and pressure gradient in the near-Earth plasma sheet. (b) Results from a computer simulation of a hybrid (KelvinHelmholtz and Rayleigh-Taylor) instability for three selected times (from Voronkov et al., 1997), showing different stages of vortex evolution.

More recently, Voronkov et al. (1997, 2000) developed a numerical model for the interaction between shear flow and ballooning mode and compared the results with largescale vortex structures in the ionosphere, associated with auroral breakups. In this model, a pressure gradient and a shear flow in the near-Earth plasma sheet, as illustrated in Fig. 13a, interact to generate an unstable hybrid mode that can rapidly grow and then saturate within tens of seconds. Possibly, this model can also explain the formation of auroral spirals. Rapid growth and saturation of the first spiral are in agreement with the model as the generation mechanism. In particular, the "detached" arc, as seen in the second spiral (e.g., at 05:39:51 UT in Fig. 3b), and the north-south aligned structure during spiral saturation, as seen in the first spiral (e.g., at 05:25:57 UT in Fig. 3a), are distinctive structures that can be found in the simulation results (Fig. 13b). For this comparison, we assume that the vorticity pattern creates a similar ionospheric footprint via field-aligned currents generated by the vorticity, as suggested by Voronkov et al. (1997). As initial parameter, two types of velocity shear flow were used, one was bidirectional and the other was (Gaussian) unidirectional. The simulation results using a unidirectional shear flow show vorticity that was closer to our observations. A unidirectional velocity profile can be expected in the transition region between both flow vortices and exists in the corresponding EIC vortices (see Fig. 8a). Addi- tionally, we showed the existence of a pressure gradient of $0.15-0.4 \mathrm{nPa} / R_{E}$ towards Earth and estimated a flow shear of several hundreds of $\mathrm{km} /\left(\mathrm{s} R_{E}\right)$ due to the flow vortices. Although the values for the observed shear flow compare favorably with the shear flow used in the model, the value used for the pressure gradient by Voronkov et al. (1997), who used $5.7 \mathrm{nPa} / R_{E}$, is one order of magnitude bigger. We note that in their later paper (Voronkov et al., 2000), they used $\sim 0.4 \mathrm{nPa} / R_{E}$ for the pressure gradient but used a bidirectional shear flow. Finally, we emphasize that the main topological difference to Hallinan's model is that in Voronkov et al.'s model the spiral would already form in the magnetosphere and simply map to the ionosphere, whereas in Hallinan's model the spiral would only form in the ionosphere (and possibly close to the ionosphere).

When discussing different theories of spiral formation care must be taken as to the type of spiral. Auroral spirals occur during different geomagnetic activities, such as quiet times and substorm times (e.g., Davis and Hallinan, 1976; Partamies et al., 2001a). Larger spirals have been associated with stronger geomagnetic activity (e.g., Partamies, 2004), and often saturate instead of unwinding to arc-like structures (Davis and Hallinan, 1976). Some studies have suggested that the auroral spiral and the auroral surge are related or even identical phenomena (e.g., Davis and Hallinan, 1976; Marklund et al., 1998) whereas others have argued that spirals are an entirely different class of auroral form (e.g., Partamies et al., 2001b). For example, Davis and Hallinan (1976) have suggested that pseudo-breakups or small substorms are in fact the development of auroral spirals, and Marklund et al. (1998) have considered auroral surges, such as the westward-traveling surge, to be only a special case of large-scale auroral spiral formations. The space signatures for our events are reminiscent of substorm and intensification (i.e. dipolarization and dispersionless particle injections), and therefore fall into the class of substorm/pseudobreakup spirals (e.g., Amm et al., 2001; Partamies et al., 2003). The spirals are distinct from those seen during quiet times, particularly, those occurring in multiples along auroral arcs and those that last for many minutes.

\section{Conclusions}

Using an unprecedented set of data sources, we have provided a description of the ionospheric and magnetospheric conditions during the development of two consecutive auroral spirals. The spirals occurred during the expansion phase of the substorm on 19 February 2008 and the subsequent intensification. The auroral spirals were of large scale $(>200 \mathrm{~km})$ and lasted less than $60 \mathrm{~s}$. They occurred in the transition region between two oppositely rotating EIC vortices, a region of strong ionospheric flows. In concert with the spirals, flow vortices, dipolarization, and ion injections occurred in the near-Earth plasma sheet at geocentric 
distance of approximately $11 R_{E}$. Although the three substorm phenomena are usually known to coexist, so far they have not been associated with auroral spirals, most likely due to a lack of ground-space coordinated observations during spiral formations.

Observations and model calculations also gave evidence of magnetic-field-aligned current generation connecting the generator region with the ionosphere during both spirals. The flow vortices were likely the source of the currents, which, in turn, were likely associated with the spiral generation. Based on timing analysis and conjugacy, we have suggested that the spirals were initiated and driven in the region between the centers of the plasma flow vortices, although the exact generation mechanism could not be identified. Two scenarios for the generation of the spirals were discussed. Either the enhanced space flow led to enhanced FACs, which caused a current sheet instability that caused the spiral in the ionosphere, or the enhanced space flow caused a shear flow instability coupled to a ballooning mode which led to the formation of the spiral in space that then mapped down to the ionosphere.

Since ground-space (plasma sheet) correlations of auroral spirals have not been reported before, this first characterization should be helpful for further numerical/theoretical investigations when identifying the generation mechanism for ground auroral spirals, at least for this active type of auroral spiral which is associated with substorms/intensifications. Another outstanding task is to investigate magnetospheric conditions during quiet-time auroral spirals and to contrast them with those presented here in order to clarify whether auroral spirals have more than one generation mechanism.

Acknowledgements. This work was supported by the NASA THEMIS project (NAS5-02099). The work of the IGEP team at the Technical University of Braunschweig was financially supported by the German Ministerium für Wirtschaft und Technologie and the German Zentrum für Luft- und Raumfahrt under grant 50QP0402. Andreas Keiling thanks Olivier Le Contel and his colleagues for their hospitality during his stay at the Centre des Environements Terrestre et Planétaires (CETP) where parts of this work were completed.

Topical Editor I. A. Daglis thanks I. Voronkov and another anonymous referee for their help in evaluating this paper.

\section{References}

Aikio, A. T., Opgenoorth, H. J., Persson, M. A. L., and Kaila, K. U.: Ground-based measurements of an arc-associated electric field, J. Atmos. Terr. Phys., 55, 797-808, 1993.

Amm, O.: Ionospheric elementary current systems in spherical coordinates and their application, J. Geomagn. Geoelectr., 49, 947955, 1997.

Amm, O. and Viljanen, A.: Ionospheric disturbance magnetic field continuation from the ground to the ionosphere using spherical elementary current systems, Earth Planets Space, 51, 431-440, 1999.

Amm, O., Janhunen, P., Kauristie, K., Opgenoorth, H. J., Pulkkinen, T. I., and Viljanen, A.: Mesoscale ionospheric electrodynamics observed with the MIRACLE network, 1, Analysis of a pseudobreakup spiral, J. Geophys. Res., 106, 24675-24690, 2001.

Angelopoulos, V.: The THEMIS mission, Space Sci. Rev., 141, 453-476, doi:10.1007/s11214-008-9336-1, 2008.

Auster, H. U., Glassmeier, K. H., Magnes, W., et al.: The THEMIS fluxgate magnetometer, Space Sci. Rev., 141, 235-264, doi:10.1007/s11214-008-9365-9, 2008.

Bester, M., Lewis, M., Roberts, B., McDonald, J., Pease, D., Thorsness, J., Frey, S., Cosgrove, D., and Rummel, D.: THEMIS operations, Space Sci. Rev., 141, 91-115, doi:10.1007/s11214008-9456-7, 2008.

Burke, W. J., Silevitch, M., and Hardy, D. A.: Observations of small-scale auroral vortices by the S3-2 satellite, J. Geophys. Res., 88, 3127-3137, 1983.

Davis, T. N. and Hallinan, T. J.: Auroral Spirals 1. Observations, J. Geophys. Res., 81, 3953-3958, 1976.

Donovan, E. F.: Modeling the magnetic effects of field-aligned currents, J. Geophys. Res., 98, 13529-13543, 1993.

Donovan, E. F., Mende, S., Jackel, B., et al.: The THEMIS all-sky imaging array-system design and initial results from the prototype imager, J. Atmos. Sol. Terr. Phys., 68, 1472-1487, 2006.

Haerendel, G.: The enigma of auroral spirals, abstract, 36th COSPAR Scientific Assembly. Held 16-23 July 2006, in Beijing, China, 2006.

Hallinan, T. J.: Auroral spirals, 2. Theory, J. Geophys. Res., 81, 3959-3965, 1976.

Keiling, A., Angelopoulos, V., Runov, A., et al.: Substorm current wedge driven by plasma flow vortices: THEMIS observations, J. Geophys. Res., 114, A00C22, doi:10.1029/2009JA014114, 2009.

Kivelson, M. G., Weygand, J. M., McPherron, R., et al.: A Preliminary Comparison Between SuperDARN Flow Vectors and Equivalent Ionospheric Currents From the GIMA, Greenland, MACCS, THEMIS, CARISMA, and CANMOS Ground Magnetometer Arrays, AGU meeting, San Francisco, USA 2009.

Küppers, F., Untiedt, J., Baumjohann, W., Lange, K., and Jones, A. G.: A two-dimensional magnetometer array for ground-based observations of auroral zone electric currents during the international magnetospheric study (IMS), J. Geophys., 46, 429-450, 1979.

Kosch, M. J., Scourfield, M. W. J., and Nielsen, E.: A selfconsistent explanation for a plasma flow vortex associated with the brightening of an auroral arc, J. Geophys. Res., 103, 29383 29391, 1998

Kosch, M. J., Amm, O., and Scourfield, M. W. J.: A plasma vortex revisited: The importance of including ionospheric conductivity measurements, J. Geophys. Res., 105, 24889-24898, 2000.

Larson, D., Lin, R., Angelopoulos, V., et al.: Solid state telescope for THEMIS, in preparation, 2009.

Lyatsky, W., Cogger, L. L., Jackel, B., Hamza, A. M., Hughes, W. J., Murr, D., and Rasmussen, O.: Substorm development as observed by Interball UV imager and 2-D magnetic array, J. Atmos. Sol. Terr. Phys., 63, 1609-1621, doi:10.1016/S1364 6826(01)00045-1, 2001.

Marklund, G. T., Karlsson, T., Blomberg, L. G., et al.: Observations of the electric field line structure associated with the westward traveling surge and large-scale auroral spirals, J. Geophys. Res., 103, 4125-4144, 1998

McFadden, J. P., Carlson, C. W., Larson, D., Angelopoulos, V., Lud- 
lam, M., Abiad, R., and Elliot, B.: The THEMIS ESA plasma instrument and in-flight calibration, Space Sci. Rev., 141, 277-302, doi:10.1107/s11214-008-9440-2, 2008.

Mende, S. B., Harris, S. E., Frey, H. U., Angelopoulos, V., Russell, C. T., Donovan, E., Jackel, B., Greffen, M., and Peticolas, L. M.: The THEMIS array of ground-based observatories for the study of auroral substorms, Space Sci. Rev., 141, 357-387, doi:10.1007/s11214-008-9380, 2008.

Opgenoorth, H. J., Pellinen, R. J., Maurer, H., Küppers, F., Heikkila, W. J., and Tanskanen, P.: Ground-based observations of an onset of localized field-aligned currents during auroral breakup around magnetic midnight, J. Geophys., 48, 101-115, 1980.

Partamies, N., Kauristie, K., Pulkkinen, T. I., and Brittnacher, M.: Statistical study of auroral spirals, J. Geophys. Res., 106, 1541515428, 2001a.

Partamies, N., Freeman, M. P., and Kauristie, K.: On the winding of auroral spirals: Interhemispheric observations and Hallinan's theory revisited, J. Geophys. Res., 106, 28913-28924, 2001 b.

Partamies, N., Amm, O., Kauristie, K., et al.: A pseudobreakup observation: Localized current wedge across the postmidnight auroral oval, J. Geophys. Res., 108(A1), A1, doi:10.1029/2002JA009276, 2003.

Partamies, N.: Meso-scale auroral physics from ground-based observations, PhD thesis, 2004.

Partamies, N., Kauristie, K., Donovan, E., Spanswick, E., and Liou, K.: Meso-scale aurora within the expansion phase bulge, Ann. Geophys., 24, 2209-2218, 2006, http://www.ann-geophys.net/24/2209/2006/.

Russell, C. T., Chi, P. J., Dearborn, D. J., Ge, Y. S., Kuo-Tiong, B., Means, J. D., Pierce, D. R., Rowe, K. M., and Snare, R. C.: THEMIS ground-based magnetometers, Space Sci. Rev., 141, 389-412, doi:10.1007/s11214-008-9337-0, 2008.
Sibeck, D. G. and Angelopoulos, V.: THEMIS science objectives and mission phases, Space Sci. Rev., 141, 35-59, doi:10.1007/s11214-008-9393-5, 2008.

Spanswick, E., Donovan, E., Friedel, R., and Korth, A.: Ground based identification of dispersionless electron injections, Geophys. Res. Lett., 34, L03101, doi:10.1029/2006GL028329, 2007.

Steen, A. and Collis, P. N.: High-time resolution imaging of auroral arc deformation at substorm onset, Planet. Space Sci., 36, 715732, 1988.

Tsyganenko, N. A.: Modeling the Earth's magnetospheric magnetic field confined within a realistic magnetopause, J. Geophys. Res., 100, 5599-5612, doi:10.1029/94JA03193, 1995.

Untiedt, J. and Baumjohann, W.: Studies of polar current systems using the IMS Scandinavian magnetometer array, Space Sci. Rev., 63, 245-390, doi:10.1007/BF0075077, 1993.

Untiedt, J., Pellinen, R., Kueppers, F., et al.: Observations of the initial development of an auroral and magnetic substorm at magnetic midnight, J. Geophys., 45, 41-56, 1978.

Voronkov, I., Rankin, R., Frycz, P., et al.: Coupling of shear flow and pressure gradient instabilities, J. Geophys. Res., 102, 96399650, 1997.

Voronkov, I., Donovan, E. F., Jackel, B. J., Samson, J. C.: Largescale vortex dynamics in the evening and midnight auroral zone: Observations and simulations, J. Geophys. Res., 105, 1850518518, 2000.

Wescott, E. M., Stenbaek-Nielsen, H. C., Davis, T. N., et al.: The L $=6.6$ 0osik Barium Plasma Injection Experiment and Magnetic Storm of March 7, 1972, J. Geophys. Res., 80, 951-967, 1975.

Weygand, J. M., Amm, O., Angelopoulos, V., et al.: Equivalent ionospheric currents from the GIMA, Greenland, MACCS, and THEMIS ground magnetometer arrays, Geophys. Res. Abstr., 11, EGU2009-10968, 2009. 